\section{Preparation of Scaffolds and New Biomaterials for Health Care Industries}

\author{
Oguzhan Gunduz ${ }^{1,2^{*}}$ and Faik Nuzhet Oktarc $^{2,3}$ \\ ${ }^{1}$ Department of Metallurgy and Material Engineering, Faculty of Technology, Marmara University, Goztepe Campus, Istanbul, 34722, Turkey \\ ${ }^{2}$ Centre for Nanotechnology \& Biomaterials Applied and Research At Marmara University, Goztepe Campus, Kadikoy, Istanbul, 34722, Turkey \\ ${ }^{3}$ Department of Bioengineering, Faculty of Engineering, Marmara University, Goztepe Campus, Istanbul, 34722, Turkey
}

"Corresponding author: Oguzhan Gunduz, Department of Metallurgy and Material Engineering, Faculty of Technology, Marmara University, Goztepe Campus, Istanbul, 34722, Turkey, Tel: 02163477681 E-mail: oguzhan@marmara.edu.tr

Received date: March 3, 2014; Accepted date: March 6, 2014; Published date: March 9, 2014

Copyright: ( ) 2014. Gunduz O, et al. This is an open-access article distributed under the terms of the Creative Commons Attribution License, which permits unrestricted use, distribution, and reproduction in any medium, provided the original author and source are credited.

\section{Editorial}

The number of treated skeletal deficiencies steadily increases in a global scale. Effective ways for bone replacements and enhancement of bone formation together with research directed to find ideal biomaterials for grafting purposes, which will feature biocompatibility and production simplicity and economy, are required. Approximately 500,000 bone-grafting procedures are annually performed in the USA. Besides aging of the world population, is also a big problem sharing governmental \& private funds and incomes. William $\mathrm{H}$. Frey, an analyst for the Brookings Institution think tank, predicts the median age in Europe will increase from 37.7 years old in 2003 to 52.3 years old by 2050 while the median age of Americans will rise to only 35.4 years old. For US between 2000 and 2050, the number of older people is projected to increase by $135 \%$. Moreover, the population aged 85 and over, which is the group most likely to need health and longterm care services, is projected to increase by $350 \%$. Over this time period, the proportion of the population that is over the age of 65 will increase from $12.7 \%$ in 2000 to $20.3 \%$ in 2050 ; the proportion of the population that is age 85 and older will increase from $1.6 \%$ in 2000 to $4.8 \%$ in 2050 .

Moreover, in the light of human life expectancy up to 90 years, the improvement of health care and the increase of accidents (due to sport activities and car accidents), the need for effective and inexpensive biomaterials available to everyone, such as those produced from biologically derived HA, is in great demand.

Medical technologies benefit the lives of people in many ways. Through the use of such technologies, people can live healthier, more productive and independent lives. Many individuals who previously may have been chronically ill, disabled, or suffering chronic pain can now look forward to leading normal or close-to- normal lives.

Biomaterials are of vital importance in the medical device industry, both for the discovery and development of synthetic organs, aesthetic procedures, and personal healthcare products. Biomaterials are mainly used in engineering new products to enhance personal appearance, which challenges the aging process. Primary consumers for anti-aging products include baby boomers and working women. Baby boomers constitute the single largest age group, driving the collagen and hyaluronic acid (US\$ 4.19 billion by 2018 ) products market. This age group is the most rapidly expanding sector and the most desirous to access anti-aging treatment. The Middle aged baby boomers are more concerned about health and their physical appearance. The global market for biomaterials is expected to reach $\$ 64.7$ billion in 2015 from US\$ 25.6 billion in 2008 with a CAGR (Compound Annual Growth Rate) of $15 \%$ from 2010 to 2015 . In 2010, the orthopedic biomaterial market recorded revenues of US\$ 12 billion or $37.5 \%$ of the total global biomaterial market.

According to Navarro et al. bone and joint degenerative and inflammatory problems affect millions of people worldwide. In fact, they account for half of all chronic diseases in people over 50 years of age in developed countries. In addition, it is predicted that the percentage of persons over 50 years of age affected by bone diseases will double by 2020 .

Autogenous grafts (but still regarded as golden standard for grafting procedures) feature the problems of the limitation of the available graft area and the fact of extra surgery. Synthetic or naturally derived hydroxyapatite (HA) is the most popular biomaterial for skeletal reconstructions. HA attracts considerable interest over the past two decades due to its excellent biocompatibility. Due to the poor mechanical properties of pure HA, HA-based composite materials are indicated for loadbearing biomedical applications. HA is the most frequently used, but it does not completely match the chemical composition of bone. HA materials, which are prepared from bovine and human resources, can be very safe when all safety procedures are sufficiently followed. The latter materials are prepared by treating them with diluted $\mathrm{HCl}$ acid, which de-mineralizes and freeze-dries the tissues. However, some diseases can survive all controlled processes. Prions, for example, which can alter themselves into very dangerous incurable forms, can survive such processes. Calcination at high temperature can solve this possible disease transmission problem. The deadly prions cannot survive calcination temperatures as high as $850^{\circ} \mathrm{C}$. Moreover, sintered BHA is absolutely safe from potentially transmitted diseases like bovine spongiform encephalopathy (BSE) and others. BHA features advantages namely low production costs and high safety regarding elimination of any risk of transmitting of the fatal prion diseases.

The HA bioceramics can be synthetic or natural. The former ones are reliable but their production is usually complicate and expensive. Natural bioceramics are economical, but they can potentially bear fatal diseases, such as human immunodeficiency virus (HIV), or bovine spongiform encephalopathy (BSE) (as explained above). There are a lot of papers, which encourage the use of HA derived from natural sources, such as human, animal, and corals. It is known from the past studies Oktar and Gunduz et al. studies that high calcinations $\left(850^{\circ} \mathrm{C}\right)$ eliminates the possible cross contamination of prions which could led normally to prion diseases such as BSE and other diseases as stated at Table 1. SHA features advantages namely low production costs and high safety regarding elimination of any risk of transmitting of the fatal prion diseases as discussed before. So far there are no records discovered about BSE diseases in Turkey. 
There is not any record found in Turkey at previous and recent EU reports about the scrape disease, which is a dangerous sheep prion disease. From the literature and recent news, this disease is seen lately in Germany, UK, and in Cyprus. According to a EU report for 2005, 367 positive cases (scrapie) were seen in Cyprus, 171 cases in France, 124 cases in Greece, 197 cases in UK and 248 cases in Italy. The preventing such diseases is screening carefully all the sheep or cattle population with scientific ways. It is also strictly prohibited to import live sheep stocks to Turkey. Furthermore, since years the mad cow disease and others were never seen in many developed countries.

\begin{tabular}{|l|l|}
\hline Animal type & Phenotype (Clinical and pathological features) \\
\hline Sheep & Scrapie \\
\hline Mink & Transmissible mink encephalopathy (TME) \\
\hline Muledeer) & Chronic wasting disease (CWD \\
\hline Cow & Bovine spongiform encepholopathy (BSE) \\
\hline
\end{tabular}

Table 1: Classification of mammalian diseases.

Structurally HA material behaves very fragile. To prevent fragility some metallic or ceramic oxides, metallic powder, whiskers and fibers. Nevertheless, whiskers are not recommended in biomedical applications because of their potential carcinogenicity. Oktar et al. and Gunduz et al. had added many different reinforcement materials to various HA sources. Human dentine HA (DHA), human enamel HA (EHA), bovine HA (BHA), commercially available HA (CHA), sheep bone (SHA), sheep enamel HA, sheep dentine HA, bovine enamel HA, bovine dentine HA, chicken bone HA and turkey bone HA. Those sources were used as main matrix material and various reinforcement materials (Bioglass, Ti powder, $\mathrm{TiO}_{2}, \mathrm{Al}_{2} \mathrm{O}_{3}, \mathrm{MgO}, \mathrm{MF}_{2}, \mathrm{CaF}_{2}, \mathrm{Zr}_{2} \mathrm{O}_{3}$, $\mathrm{Yt}_{2} \mathrm{O}_{3}, \mathrm{Li}_{2} \mathrm{O}_{3}$ and many others) were added to get more load carriable bioceramic material. But from material load carrying ability commercial window glass the most suitable ones. $133 \mathrm{MPa}$ for $10 \%$ addition sintered at $1200^{\circ} \mathrm{C}$. At BHA the highest compression strength value was measured as $67 \mathrm{MPa}$, which is sintered at $1200^{\circ} \mathrm{C}$. Double compression value $(\mathrm{MPa})$ was obtained by adding reinforcement material. Some HA types have low compression value, but from cell culture side some weak HA composites had excellent values like BHALithiumoxide, EHATi, BHATi and etc.

Besides HA composites as stated above, there are also very recent techniques (with different new materials) like generating bubbles and thin fiber (electrostatic fiber formation or electro-spinning) can also help to produce some true nano-structures for health care industry. The importance of bubbles in recent times is being 'blown' to new horizons, with their promising roles crossing physical and life sciences. Today, they are finding applications and implications in numerous areas of daily interactions spanning several disciplines, including engineering, environmental, food and health care sciences. Bubbles are critically important in diagnostics (as contrast agents for ultrasound imaging) and especially in targeted therapeutics (delivery vehicles for target therapy) and also used as gas carriers for blood substitution. Conventionally, bubbles are generated in microfluidic devices at the liquid and gas interface in various micro-channel or capillary junctions. There are many types of devices, which have multiple intersections and flows, the majority of which use microfluidic principles. These devices are ideal for obtaining uniform size microbubbles and similar products since they have the advantage of continuous, reproducible and scalable production. Microfluidic technologies offer low-cost and easy-to-use platforms for controlling gas and fluid.

The primary focus of research "electrospinning and electrospraying" has been mostly the fundamental physics underlying the process and its application in biomedical engineering for constructing wound dressings and tissue scaffolds for organ therapy. Electrospinning and Electrospraying have emerged in recent years as a popular choice for producing continuous threads, particles, rods, fiber arrays and nonwoven fabrics from a range of materials including polymers, ceramics, composites and food-grade materials with a range of diameters (1-100 $\mu \mathrm{m})$. Electrospun fibres at this scale have a number of desirable characteristics such as very large surface area to volume ratio, flexibility to incorporate surface functionalities, and superior mechanical properties (e.g. stiffness and tensile strength).

According to nanotechnology for health care report (July 2008: Darren Bhattachary, Richard Stockley and Andrew Hunter, BMRB, UK) has the potential to make a significant impact on health care by delivering step-changes in disease diagnosis and monitoring, implants and regenerative medicine, drug delivery, as well as research tools for drug discovery and biomedical science. Karwal et al. had described that nanotechnology is the engineering of functional systems at the molecular scale and nanotechnology enhanced materials will enable a weight reduction accompanied by an increase in stability and an improved functionality. Traditionally nanotechnology in pharmacy has been associated with drug delivery, where the size of the delivery vehicle, whether it be a liposome, a polymer or even a metallic nanoparticle and its consequent ability to evade many of our bodies' natural defenses has been the main attraction. Electrospraying, electrospinning and bubbling methods are the most used popular methods, which are encountered within the unlimited limits of the technology of 21 st century - Nanotechnology.

There are also some different applications of nanotechnologies at a new biomaterials like nanobioceramic production from natural calcite and aragonite structures such as from different sea originated sources like: sea urchin shells, sea snail shells, mussel shells, cuttle fish (Sepia officinalis) and corals. There are also possibility to produce nanobioceramics from land originated natural calcite and aragonite structures such as chicken egg shells, ostrich egg shells and land snail shells. First HA production in the English literature was invented by Rocha et al, which the idea was given by Oktar et al. Now, there are over 40 scientific papers in the internet and was cited over 56 times. The second work by the same group (including also Oktar )(Lemos et al.) (HA production from sea shells) was conducted and cited 42 times. Those two productions were made hydrothermally under high pressure. Besim et al. had conducted such important studies about HA production with pressurized hydrothermal method from corals.

HA coatings on medical implants deposited by conventional thermal plasma spraying function as an intermediate layer between human tissues and the metallic implant. However, this method supplies very thick films, which might crack, peel or dissolve in biological fluids. These shortcomings supported the use of alternative coating techniques for the deposition of films, such as liquid plasma spraying, radio-frequency and direct current magnetron sputtering, ion implantation, ion beam sputtering, pulsed laser deposition (PLD) or combination of different technique. Oktar et al. had developed new type of HA coatings with PLD method and had got very successful results (normally HA plasma coatings are around 100-120 $\mu \mathrm{m}$ and 
Citation: Oguzhan Gunduz, Faik Nuzhet Oktarc (2014) Preparation of Scaffolds and New Biomaterials for Health Care Industries. Bioceram Dev

Page 3 of 3

PLD are about 5-10 $\mu \mathrm{m}$ thick. These make PLD coatings very successful for implant coatings but those are still in investigation.

Lately Prof. Oktar and Dr. Gunduz had worked with well organized groups world wide (Japan, Australia, Greece, UK, Romania) MSc students (Kel, Tuyel) and co-workers (i.e. Ben-Nissan, Ozyegin, Chou, Macha, Tămăşan, Turner, Ahmad, Agaogulları, Gokce, Agathopoulos, Samur, Sima, Ristoscu) producing nano-bioceramic powders from various sea creatures (sea shells, sea snail etc.).

Dr. Oguzhan Gunduz, one of founders, has served as a member of board for Centre of Nanotechnology \& Biomaterials Application and Research at Marmara University. He has gained his $\mathrm{PhD}$ from the University College London, UK. He has actively pursued novel advanced material processing and forming research, publishing over 50 papers. He is engaged in internationally leading research addressing some of the global healthcare challenges.
Prof. Faik Nuzhet Oktar one of founders, has served as a member of board for Centre of Nanotechnology \& Biomaterials Application and Research at Marmara University. He was graduated from University of Marmara (BS Degree), School of Dentistry in Istanbul, Turkey. He had his Master of Science (MSc) and $\mathrm{PhD}$ degree from University of Bosporus from Biomedical Engineering Institute, Istanbul, Turkey. His research interests covers plasma spraying process of HA on titanium, bioactivity studies, porous HA and bioceramic structures, HA composites, nanomaterials, nano-biomaterials, drug delivery, biocompatibility testing, PLD deposition, according to web of science Prof. Dr. Faik Nuzhet Oktar has 104 publications which accorded as primary indexes as SCI, SCI-expanded and other primary indexes. He has $\mathrm{H}$ factor (according the site web of science) as 14 and have about 700 citations. 